\title{
Forensic Discrimination of Sheet Glass by a Refractive-Index Measurement and Elemental Analysis with Synchrotron Radiation X-ray Fluorescence Spectrometry
}

\author{
Yasuhiro SuZukI,* Masaaki KaSamatsu,* Shinichi SuZUKI,* Toshio NaKanISHI,** \\ Masahisa Takatsu, ** Seiji Muratsu, $* *$ Osamu Shimoda, ** Seiya Watanabe, $* *$ \\ Yoshinori NiSHIWAKI, $* *$ and Naoki MIYAMOTO** \\ *National Research Institute of Police Science, 6-3-1, Kashiwanoha, Kashiwa, Chiba 277-0882, Japan \\ **Forensic Science Laboratory, Hyogo Prefectural Police H. Q., 5-4-1, Shimoyamate-dori, \\ Chuo-ku, Kobe 650-8510, Japan
}

\begin{abstract}
Measurements of the refractive index (RI) and elemental analysis using synchrotron radiation X-ray fluorescence spectrometry (SR-XRF) were applied to the forensic discrimination of sheet-glass samples from different origins. The refractive index was calculated from the matching temperature at which the glass fragments became invisible in silicone oil. Fragments smaller than $1 \mathrm{~mm}$ in maximum diameter were taken from each of 11 sheet glasses and subjected to analysis by SR-XRF. The XRF spectrum of these samples indicated that a comparison of 6 elements $(\mathrm{Ca}, \mathrm{Fe}, \mathrm{Sr}, \mathrm{Zr}, \mathrm{Ba}$ and $\mathrm{Ce}$ ) was useful for the discrimination of sheet glasses. Cluster analysis was performed using 33 sets of SR-XRF data obtained by triplicate measurements for the 11 glasses. Comparing 528 pairs among 33 samples, 515 pairs could be correctly discriminated. The number of indistinguishable pairs could be reduced from 36 to 4 by comparing the SR-XRF data. Elemental analysis by SR-XRF could provide small glass fragments with a more evidential value than the solely measurement of only RI, through a significant improvement of the discrimination capability.
\end{abstract}

(Received February 18, 2005; Accepted April 28, 2005)

\section{Introduction}

A glass fragment produced upon a destruction of a sheet glass is one of the most important items of physical evidence encountered in forensic science laboratories frequently. When a burglar enteres a house after breaking a window pane, tiny glass fragments can be transferred from the window to the clothes or the body of the burglar. In an automobile hit-and-run case, glass fragments found at a crime scene are compared with those taken from the windshield and the side window of a suspected car. If a series of scientific examinations prove a similarity between the glass fragments at a crime scene and those from a suspect, they can provide valuable physical evidence to associate the suspect with the crime. ${ }^{1-3}$

The forensic discrimination of glass fragments has been carried out by comparing the color and thickness, ${ }^{4}$ observing the original surface under an interference microscope, ${ }^{5}$ and measuring the refractive index (RI) and density. ${ }^{6-10}$ However, a comparison of the optical and morphological characteristics can be applied only to sufficiently large fragments, which are rarely found in actual cases. Improvements in quality control during the manufacturing of glass have made the variation for RI of glass smaller, resulting in a reduced discrimination capability by the comparison of RI. ${ }^{11}$

In order to increase the evidential value of glass fragments by enhancing the discrimination capability, the analysis and comparison of elemental compositions have been investigated for the further discrimination of glass fragments using instrumental analysis, such as neutron activation analysis, ${ }^{12}$ scanning electron microscopy/energy dispersive X-ray fluorescence, ${ }^{13}$ X-ray fluorescence spectrometry (XRF), ${ }^{14-16}$ atomic absorption spectrometry, ${ }^{15}$ inductively coupled plasma atomic emission, ${ }^{14,17,18}$ and inductively coupled plasma mass spectrometry (ICP-MS). ${ }^{19-23}$ Among these procedures, ICP-MS is considered to be the most suitable for forensic glass examination, because it can offer high sensitivity indispensable for the analysis of trace impurities, the comparison of which is effective for the discrimination of various types of evidential materials. However, the application of ICP-MS to evidential glass is limited by its disadvantages, such as large amount of sample $(2-10 \mathrm{mg})$ required, destruction of fragments, complex and time-consuming pretreatment to dissolve glass into acid solution. The nondestructive analysis of small fragments using an EPMA is not suitable for the detection of trace elements in glass because of its low sensitivity.

Synchrotron radiation X-ray fluorescence spectrometry (SR$\mathrm{XRF}$ ) is an analytical technique, which uses brilliant monochromatic X-rays as an excitation source. The extremely high sensitivity and small beam size of SR-XRF can provide a potential for the nondestructive analysis of trace elements in a small sample, such as glass fragments. Actually, Nakai et al. have reported the successful application of SR-XRF to geological and biological samples. ${ }^{24-26}$ In a previous study, ${ }^{21}$ the authors examined the analysis of trace impurities in headlight glass using the SR-XRF and ICP-MS and demonstrated that SRXRF could be an alternative method of ICP-MS based on the agreement of the analytical results obtained by these methods. In the present study, the RI measurement and trace elemental analysis with SR-XRF were applied to the forensic 
Table 1 Sheet glass samples collected for the experiments

\begin{tabular}{|c|c|c|c|c|c|c|c|}
\hline Sample No. & Manufacturer & Plant & Furnace & Float/Figured & Color/Style & Thickness/mm & Use \\
\hline 1 & A & Kansai & Figured-Float & Figured & —a $^{\mathrm{a}}$ & 4.0 & Building \\
\hline 2 & A & Kansai & Figured-Float & Figured & - $^{\mathrm{a}}$ & 6.0 & Building \\
\hline 3 & A & Keihin & Float No.1 & Float & Clear & 6.0 & Building \\
\hline 4 & A & Aichi & Float No.2 & Float & Brown & 3.5 & Car \\
\hline 5 & $\mathrm{~A}$ & Aichi & Float No.2 & Float & gray & 3.5 & Car \\
\hline 6 & $\mathrm{C}$ & Matsuzaka & Float No.2 & Float & Green & 3.5 & Car \\
\hline 7 & $\mathrm{C}$ & Matsuzaka & Float No.1 & Float & Blue & 3.5 & Car \\
\hline 8 & $\mathrm{C}$ & Matsuzaka & Float No.1 & Float & Green & 3.5 & Car \\
\hline 9 & $\mathrm{C}$ & Matsuzaka & Float No.1 & Float & New green & 3.5 & Car \\
\hline 10 & $\mathrm{~N}$ & Maizuru & M3F & Float & Gray & 3.5 & Car \\
\hline 11 & $\mathrm{~N}$ & Maizuru & M3F & Float & Bronze & 3.5 & Car \\
\hline
\end{tabular}

a. Colorless but not clear due to the design on the surface.

discrimination of sheet-glass samples from different sources. Cluster analysis was employed to the interpretation of multielemental data obtained by SR-XRF. The improvement of the discrimination capability by combing these two methods was also estimated in terms of the number of indistinguishable pairs.

\section{Experimental}

\section{Instruments}

The RI measurement system used in this study was a phasecontrast microscope with a hot stage of Model FP82HT (METTLER, Greifensee, Switzerland) and a control unit of Model GRIM2 (foster + freeman, Worcestershire, UK) attached. As illumination for the RI measurement, a halogen bulb lamp was used together with an interference filter of $589 \mathrm{~nm}$.

The SR-XRF experiments were carried out at Super Photon Ring $8 \mathrm{GeV}$ (SPring-8), Nishi-Harima, Hyogo, Japan. Monochromatic X-rays of $75 \mathrm{keV}$ from the BL37XU beam line were used as an excitation source to irradiate a glass fragment after passing through an ion chamber to monitor the X-ray intensity. The beam size was adjusted to $1.0 \mathrm{~mm} \times 0.5 \mathrm{~mm}$ by a combination of horizontal and vertical slits. The SR-XRF spectrum was measured using a pure-Ge solid state detector from CANBERA (Connecticut, USA). The geometry between the X-ray beam and the detector was fixed at $90^{\circ}$. All of the measurements were performed under atmospheric conditions. The accumulation time was set at $1000 \mathrm{~s}$.

\section{Chemicals and samples}

Silicone oil (SH710) was purchased from Toray·Dow Corning Silicone (Tokyo, Japan) for RI measurements. Standard glass samples (K1, K7, BK7, K5 and PK3) with certified RI values by Ohara Inc. (Kanagawa, Japan) were used to make a calibration curve between RI of silicone oil and the temperature. NIST Standard Reference Material 612 (trace elements in glass) was used to determine the detection limit by SR-XRF. Eleven kinds of different sheet-glass samples, whose information is summarized in Table 1, were collected in order to estimate the discrimination capability of the present method. Purified water prepared by a Milli-Q system (Nihon Millipore Kogyo Ltd., Tokyo, Japan), ethanol and acetone of reagent grade (Wako Pure Chemical Ltd., Osaka, Japan) were used to wash glass samples as well as handling tools for sample preparation. Sheet glasses were washed with water, dried in the air and broken with a hammer into small pieces $(<10 \mathrm{~mm})$ wrapped in a sheet of paper. Among these pieces of sheet glass, 4 pieces were taken from different positions in each sample. One piece was
Table 2 Results of the RI measurement for the sheet glass

\begin{tabular}{cccc}
\hline Sample & RI & Sample & RI \\
\hline 1 & 1.5195 & 7 & 1.5186 \\
2 & 1.5196 & 8 & 1.5188 \\
3 & 1.5187 & 9 & 1.5206 \\
4 & 1.5203 & 10 & 1.5202 \\
5 & 1.5204 & 11 & 1.5203 \\
6 & 1.5193 & & \\
\hline
\end{tabular}

used for an RI measurement, and the other 3 were for elemental analysis with SR-XRF.

\section{Procedures}

Refractive index measurement. A piece of sheet glass was crushed into an appropriate size (approximately $0.5 \mathrm{~mm}$ or smaller) with an agate mortar after washing with ethanol and then dried in the air. These fragments were mounted in silicone oil and observed with a microscope as the temperature of the hot stage was varied at $4^{\circ} \mathrm{C} / \mathrm{min}$. The match temperature at which the glass became invisible due to the same RI with the oil was measured. The refractive index of the glass was calculated using the match temperature and the calibration curve. The measurement was repeated 5 times for each sample using different fragments. Glass fragments containing the original surface were excluded from the RI measurement, because the surface of the sheet glass could exhibit RI values different from that of bulk glass. ${ }^{9,27}$

Analysis of trace elements by SR-XRF. Each piece of sheet glass was washed 6 times with acetone and water. After being dried in an oven, each piece was crushed into fragments of appropriate size with the agate mortar. A fragment smaller than $1 \mathrm{~mm}$ in maximum diameter was selected from each piece. This fragment was wrapped with thin film ( $6 \mu \mathrm{m}$, polypropylene), attached to a sample holder, as shown in Fig. 1, and used for elemental analysis with SR-XRF.

\section{Results and Discussion}

\section{Results of RI measurements}

The results of RI measurements for the 11 sheet glasses are given in Table 2. It is reported that the variation of RI within a sheet glass across different sampling positions is smaller than 0.0002 , which was calculated as the maximum range of the mean \pm 2 SD. ${ }^{9,14}$ Compared with the inner-sample variation, RI 


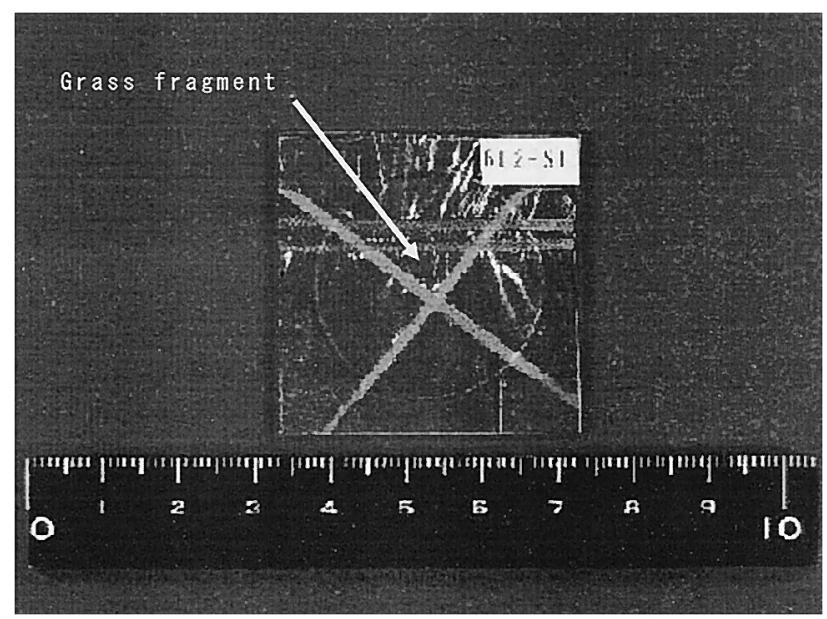

Fig. 1 Glass fragment attached to a sample holder.

for the 11 sheet glasses occurred over a wide range from 1.5186 to 1.5204. Discrimination of these glasses was performed as follows. A pair of sheet glasses is considered to be distinguishable when their RI differs by more than 0.0002 . On the contrary, a pair of sheet glasses is considered to be indistinguishable when the difference in RI is equal to or less than this criterion. The difference in the RI value was calculated for all of 66 pairs among 11 samples. As a result, 54 pairs $(82 \%)$ were distinguishable, whereas 12 pairs $(18 \%)$ were not. This result may not represent a general trend, because of the limited number of samples examined. Actually, the rate of indistinguishable pairs was under $10 \%$ in the previous studies carried out using a sufficiently large number of glass samples. However, it should be remembered that discrimination only by the RI comparison always runs a risk of type-II errors, ${ }^{14}$ which means incorrect attribution of two samples to a common origin. This opinion is supported by the fact that 8 of 12 indistinguishable pairs are produced by different manufacturers.

\section{Elemental analysis using $S R-X R F$}

In order to select useful elements for the forensic comparison of sheet glasses, the XRF spectrum for 11 different samples in Table 1 were measured and compared with each other. The measurement was repeated three times using different fragments taken from each sample separately. The representative spectra of samples 3 and 7 are shown in Figs. 2(a) and 2(b), respectively, as an example of successful discrimination by elemental analysis. These two samples could not be distinguished by a comparison of the RI, because the difference in RI between the two was smaller than the variation in the same sample. However, a significant difference was observed in the XRF spectra of these samples. The peaks of Ce $K_{\alpha}$ and Ce $K_{\beta}$ were detected only from sample 3 . The peak intensity of Fe $K_{\alpha}$ was higher than that of $\mathrm{Ca} K_{\alpha}$ in sample 7 , while the former was lower than the latter in sample 3. All 66 pairs among 11 samples were compared in a similar manner. As a result, 6 elements $(\mathrm{Ca}, \mathrm{Fe}, \mathrm{Sr}, \mathrm{Zr}, \mathrm{Ba}$ and $\mathrm{Ce})$ were found to be useful for the discrimination of sheet glasses. It should be emphasized that excitation by high-energy X-rays of $75 \mathrm{keV}$ could make it possible to analyze $\mathrm{Ba}$ and $\mathrm{Ce}$ using the $\mathrm{K}$ lines of these elements with no interference from the major elements. Analysis of these heavy elements in glass using the L lines were interfered by an overlapping of the $\mathrm{K}$ lines from major elements, such as $\mathrm{Si}$ and $\mathrm{Ca}$, when low-energy X-rays under 30
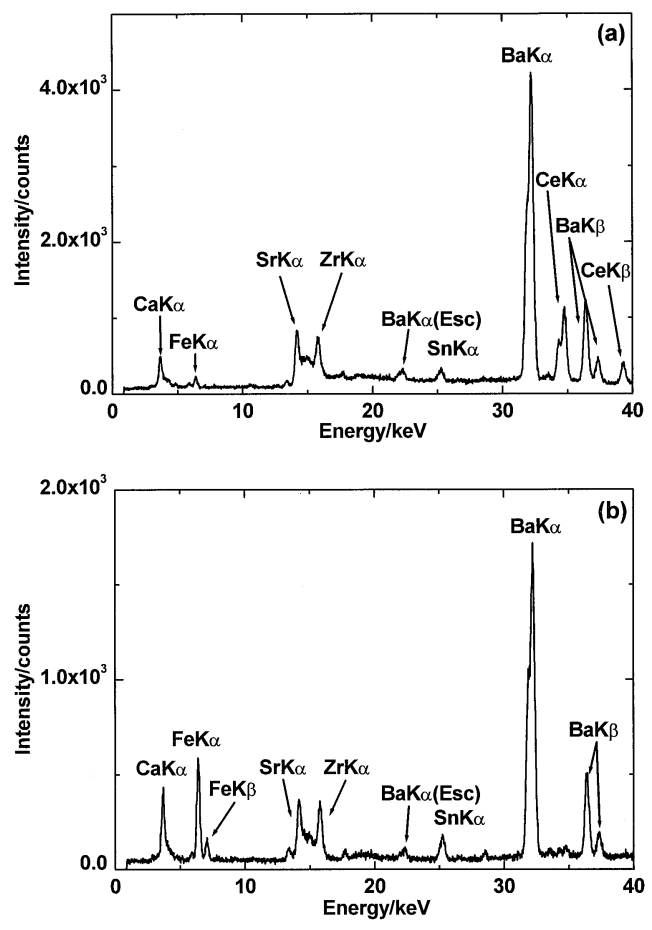

Fig. 2 X-ray fluorescence spectrum of (a) sample 3 and (b) sample 7.

$\mathrm{keV}$ were employed as an excitation source.

The X-ray intensities of 6 elements measured for the 11 sheet glasses are summarized in Table 3. Fragments taken from NIST standard reference material were analyzed, and the detection limits (DL) for 3 elements ( $\mathrm{Sr}, \mathrm{Ba}$ and $\mathrm{Ce}$ ) with certified or reference value were calculated according to the expression $3 B^{1 / 2}(C / N)$, where $B$ is the background counts, $N$ the net counts, and $C$ the content of the element. ${ }^{28}$

\section{Discrimination of sheet glasses by cluster analysis}

Cluster analysis was performed using 33 sets of SR-XRF data in Table 3, which were obtained by triplicate measurements for each of the 11 sheet glasses. In order to calculate the Euclidian distance between the data, 4 parameters (the intensity ratios, such as $\mathrm{Ca} K_{\alpha} / \mathrm{Fe} K_{\alpha}, \mathrm{Sr} K_{\alpha} / \mathrm{Ba} K_{\alpha}, \mathrm{Zr} K_{\alpha} / \mathrm{Fe} K_{\alpha}$, and Ce $K_{\alpha} / \mathrm{Ba}$ $K_{\alpha}$, were used as variances. The obtained dendrogram is shown in Fig. 3.

The number of pairs among the 33 samples in the dendrogram was $528\left(={ }_{33} \mathrm{C}_{2}\right)$. These 528 pairs could be classified into 33 (= $\left.{ }_{3} \mathrm{C}_{2} \times 11\right)$ pairs within the same glass and $495\left(=3 \times 3 \times{ }_{11} \mathrm{C}_{2}\right)$ pairs between different ones. The discrimination of the 528 pairs was performed as follows. A pair of samples were considered to be distinguishable when they make a cluster at a similarity lower than 0.922 , whereas they were considered to be indistinguishable and to originate from a same source when they were classified into a same cluster at a similarity over this criterion, because most of samples from a same source make a cluster under this value. The discrimination results are summarized in Table 4. Out of 33 pairs within a same sample, 25 were correctly recognized, and the other 8 were incorrectly discriminated. Out of 495 pairs between different samples, 490 were correctly distinguished and the other 5 were not. The rate of correct discrimination as a whole was 515 out of 528 (97.5\%), which is much better than that by only RI. It should also be emphasized that 32 out of 36 pairs with indistinguishable RI values could be discriminated by 
Table 3 Analytical results for sheet-glass samples by SR-XRF

\begin{tabular}{|c|c|c|c|c|c|c|}
\hline \multirow{2}{*}{$\begin{array}{c}\text { Sample } \\
\text { (Measurement } \\
\text { number) }\end{array}$} & \multicolumn{6}{|c|}{ X-ray intensity (peak area)/count } \\
\hline & $\mathrm{Ca} K_{\alpha}$ & $\mathrm{Fe} K_{\alpha}$ & $\operatorname{Sr} K_{\alpha}$ & $\operatorname{Zr} K_{\alpha}$ & Ва $K_{\alpha}$ & Ce $K_{\alpha}$ \\
\hline $1(1)$ & $7.97 \times 10^{3}$ & $2.87 \times 10^{3}$ & $3.66 \times 10^{3}$ & $5.65 \times 10^{3}$ & $5.12 \times 10^{4}$ & $1.89 \times 10^{3}$ \\
\hline $1(2)$ & $6.22 \times 10^{3}$ & $2.12 \times 10^{3}$ & $1.87 \times 10^{3}$ & $3.01 \times 10^{3}$ & $2.90 \times 10^{4}$ & $8.56 \times 10^{2}$ \\
\hline $1(3)$ & $5.18 \times 10^{3}$ & $2.16 \times 10^{3}$ & $2.30 \times 10^{3}$ & $3.00 \times 10^{3}$ & $3.12 \times 10^{4}$ & $1.22 \times 10^{3}$ \\
\hline $2(1)$ & $6.65 \times 10^{3}$ & $2.11 \times 10^{3}$ & $2.60 \times 10^{3}$ & $3.78 \times 10^{3}$ & $4.56 \times 10^{4}$ & $1.87 \times 10^{3}$ \\
\hline $2(2)$ & $9.19 \times 10^{3}$ & $2.60 \times 10^{3}$ & $4.65 \times 10^{3}$ & $7.70 \times 10^{3}$ & $9.14 \times 10^{4}$ & $2.43 \times 10^{3}$ \\
\hline $2(3)$ & $6.21 \times 10^{3}$ & $2.03 \times 10^{3}$ & $2.71 \times 10^{3}$ & $4.02 \times 10^{3}$ & $4.95 \times 10^{4}$ & $1.75 \times 10^{3}$ \\
\hline $3(1)$ & $5.96 \times 10^{3}$ & $1.48 \times 10^{3}$ & $6.46 \times 10^{3}$ & $7.73 \times 10^{3}$ & $1.17 \times 10^{5}$ & $2.69 \times 10^{4}$ \\
\hline $3(2)$ & $7.28 \times 10^{3}$ & $1.53 \times 10^{3}$ & $4.52 \times 10^{3}$ & $3.27 \times 10^{3}$ & $5.11 \times 10^{4}$ & $1.12 \times 10^{4}$ \\
\hline $3(3)$ & $5.71 \times 10^{3}$ & $1.25 \times 10^{3}$ & $6.58 \times 10^{3}$ & $3.82 \times 10^{3}$ & $5.55 \times 10^{4}$ & $1.28 \times 10^{4}$ \\
\hline $4(1)$ & $5.49 \times 10^{3}$ & $3.71 \times 10^{3}$ & $1.63 \times 10^{3}$ & $3.04 \times 10^{3}$ & $2.95 \times 10^{4}$ & $2.30 \times 10^{4}$ \\
\hline $4(2)$ & $6.86 \times 10^{3}$ & $4.84 \times 10^{3}$ & $2.98 \times 10^{3}$ & $5.47 \times 10^{3}$ & $5.82 \times 10^{4}$ & $4.25 \times 10^{4}$ \\
\hline $4(3)$ & $4.70 \times 10^{3}$ & $3.41 \times 10^{3}$ & $2.36 \times 10^{3}$ & $3.37 \times 10^{3}$ & $3.75 \times 10^{4}$ & $2.65 \times 10^{4}$ \\
\hline $5(1)$ & $6.22 \times 10^{3}$ & $3.02 \times 10^{3}$ & $2.14 \times 10^{3}$ & $5.26 \times 10^{3}$ & $4.88 \times 10^{4}$ & $1.53 \times 10^{4}$ \\
\hline $5(2)$ & $5.44 \times 10^{3}$ & $2.38 \times 10^{3}$ & $1.80 \times 10^{3}$ & $3.58 \times 10^{3}$ & $3.83 \times 10^{4}$ & $1.05 \times 10^{4}$ \\
\hline $5(3)$ & $5.17 \times 10^{3}$ & $2.36 \times 10^{3}$ & $2.50 \times 10^{3}$ & $4.60 \times 10^{3}$ & $5.76 \times 10^{4}$ & $1.52 \times 10^{4}$ \\
\hline $6(1)$ & $8.24 \times 10^{3}$ & $1.56 \times 10^{4}$ & $3.76 \times 10^{3}$ & $9.44 \times 10^{3}$ & $8.02 \times 10^{4}$ & - $^{\mathrm{a}}$ \\
\hline $6(2)$ & $1.11 \times 10^{4}$ & $1.82 \times 10^{4}$ & $4.97 \times 10^{4}$ & $7.72 \times 10^{4}$ & $8.05 \times 10^{4}$ & - \\
\hline $6(3)$ & $3.27 \times 10^{3}$ & $7.24 \times 10^{3}$ & $2.39 \times 10^{3}$ & $2.63 \times 10^{3}$ & $2.45 \times 10^{4}$ & - \\
\hline $7(1)$ & $6.14 \times 10^{3}$ & $7.01 \times 10^{3}$ & $3.41 \times 10^{3}$ & $3.30 \times 10^{3}$ & $4.47 \times 10^{4}$ & - \\
\hline $7(2)$ & $6.12 \times 10^{3}$ & $8.19 \times 10^{3}$ & $3.79 \times 10^{3}$ & $4.45 \times 10^{3}$ & $4.74 \times 10^{4}$ & - \\
\hline $7(3)$ & $5.34 \times 10^{3}$ & $7.42 \times 10^{3}$ & $3.69 \times 10^{3}$ & $3.43 \times 10^{3}$ & $3.71 \times 10^{4}$ & - \\
\hline $8(1)$ & $3.32 \times 10^{3}$ & $8.96 \times 10^{3}$ & $3.18 \times 10^{3}$ & $2.99 \times 10^{3}$ & $4.84 \times 10^{4}$ & $4.54 \times 10^{4}$ \\
\hline $8(2)$ & $4.01 \times 10^{3}$ & $8.48 \times 10^{3}$ & $2.38 \times 10^{3}$ & $2.17 \times 10^{3}$ & $4.33 \times 10^{4}$ & $3.92 \times 10^{4}$ \\
\hline $8(3)$ & $6.30 \times 10^{3}$ & $1.38 \times 10^{4}$ & $2.41 \times 10^{3}$ & $2.98 \times 10^{3}$ & $6.32 \times 10^{4}$ & $5.99 \times 10^{4}$ \\
\hline $9(1)$ & $3.93 \times 10^{3}$ & $1.26 \times 10^{4}$ & $1.67 \times 10^{3}$ & $1.66 \times 10^{3}$ & $3.58 \times 10^{4}$ & $1.52 \times 10^{5}$ \\
\hline $9(2)$ & $3.62 \times 10^{3}$ & $1.03 \times 10^{4}$ & $1.02 \times 10^{3}$ & $1.27 \times 10^{3}$ & $1.89 \times 10^{4}$ & $7.78 \times 10^{4}$ \\
\hline $9(3)$ & $3.20 \times 10^{3}$ & $9.19 \times 10^{3}$ & $1.89 \times 10^{3}$ & $1.19 \times 10^{3}$ & $2.21 \times 10^{4}$ & $8.97 \times 10^{4}$ \\
\hline $10(1)$ & $3.37 \times 10^{3}$ & $1.35 \times 10^{3}$ & $7.10 \times 10^{2}$ & $2.73 \times 10^{2}$ & $5.51 \times 10^{3}$ & - \\
\hline $10(2)$ & $5.50 \times 10^{3}$ & $2.85 \times 10^{3}$ & $1.96 \times 10^{3}$ & $1.66 \times 10^{3}$ & $2.00 \times 10^{4}$ & - \\
\hline $10(3)$ & $3.28 \times 10^{3}$ & $1.62 \times 10^{3}$ & $9.10 \times 10^{2}$ & $1.05 \times 10^{3}$ & $1.09 \times 10^{4}$ & - \\
\hline $11(1)$ & $5.83 \times 10^{3}$ & $4.04 \times 10^{3}$ & $1.71 \times 10^{3}$ & $9.94 \times 10^{2}$ & $2.01 \times 10^{4}$ & $4.72 \times 10^{4}$ \\
\hline $11(2)$ & $5.76 \times 10^{3}$ & $3.70 \times 10^{3}$ & $1.51 \times 10^{3}$ & $6.60 \times 10^{2}$ & $1.72 \times 10^{4}$ & $3.96 \times 10^{4}$ \\
\hline $11(3)$ & $5.06 \times 10^{3}$ & $3.73 \times 10^{3}$ & $1.90 \times 10^{3}$ & $7.75 \times 10^{2}$ & $2.19 \times 10^{4}$ & $4.73 \times 10^{4}$ \\
\hline \multirow{3}{*}{\multicolumn{2}{|c|}{$\begin{array}{l}\text { NIST } 612 \\
\text { Certified value/ } \mu \mathrm{g} \mathrm{g}^{-1} \\
\mathrm{DL} / \mu \mathrm{g} \mathrm{g}^{-1 \mathrm{~b}}\end{array}$}} & \multicolumn{3}{|c|}{$2.40 \times 10^{4}$} & $1.16 \times 10^{5}$ & $1.27 \times 10^{5}$ \\
\hline & & \multicolumn{3}{|c|}{$78.4 \pm 0.2$} & $(41)^{c}$ & $(39)^{\mathrm{c}}$ \\
\hline & & \multicolumn{3}{|c|}{5.6} & 2.5 & 2.2 \\
\hline
\end{tabular}

a. - : Not detected. b. Detection limit. c. Reference value.

Table 4 Discrimination results for sheet-glass samples by cluster analysis of SR-XRF data

\begin{tabular}{cccc}
\hline \multirow{2}{*}{ Source } & \multicolumn{2}{c}{ Discrimination result } & \multirow{2}{*}{ Total } \\
\cline { 2 - 3 } & Ditinguishable & Indistinguishable & \\
\hline Same & 8 & 25 & 33 \\
Different & 490 & 5 & 495 \\
Total & 498 & 30 & 528 \\
\hline
\end{tabular}

comparing the SR-XRF data. The only 4 pairs that could not be distinguished by SR-XRF were those between samples 1(1), 1(2) and sample 2(1), 2(3). All of these pairs were produced at a common plant of a manufacturer with the same method. These results demonstrate that the analysis of elemental compositions by SR-XRF can provide small glass fragments with a greater evidential value than the sole measurement of RI, through a significant improvement of the discrimination capability.

\section{Acknowledgements}

The authors are deeply grateful to Dr. Yasuko Terada at the Japan Synchrotron Radiation Research Institute for her valuable support in the experiments at SPring-8.

\section{References}

1. R. Saferstein, "Criminalistics, An Introduction to Forensic Science", 1995, Prentice-Hill, New Jersey.

2. S. Suzuki and S. Seta, Bunseki, 1996, 253, 44.

3. Y. Suzuki, J. Plasma Fusion Res., 2002, 78, 659.

4. J. M. Curran, T. N. Hicks, and J. S. Buckleton, "Forensic Interpretation of Glass Evidence", 2000, CRC Press, Florida.

5. J. Locke and J. A. Zoro, Forensic Sci. Int., 1983, 22, 221.

6. J. Locke and M. Underhill, Forensic Sci. Int., 1985, 27, 247.

7. R. Sugita, Y. Suzuki, S. Suzuki, and Y. Marumo, Jpn. J. Sci. Tech. Iden., 1997, 2, 89.

8. R. Sugita, Y. Suzuki, S. Suzuki, and Y. Marumo, Report of 


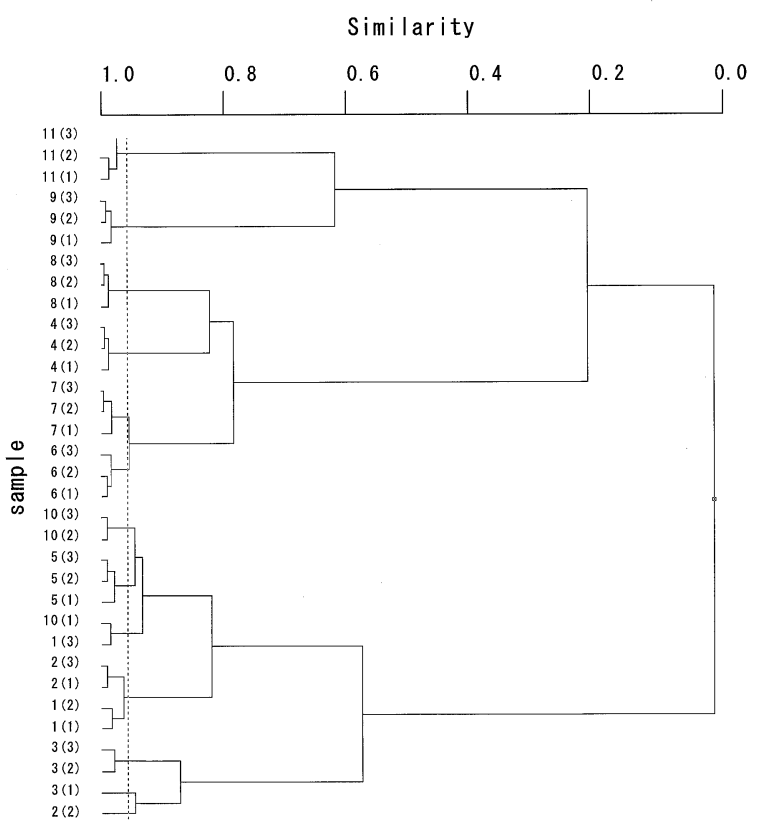

Fig. 3 Dendrogram of 11 sheet glasses using the analytical results by SR-XRF. The dotted line in the dendrogram shows a similarity of 0.922 .

the National Research Institute of Police Science (Research on Forensic Science), 1998, 51, 84.

9. Y. Suzuki, M. Kasamatsu, R. Sugita, S. Suzuki, and Y. Marumo, Jpn. J. Sci. Tech. Iden., 2001, 5, 85.

10. A. W. N. Newton, J. M. Curran, C. M. Triggs, and J. S. Buckleton, Forensic Sci. Int., 2004, 140, 185.

11. R. D. Koons and J. Buscaglia, Forensic Sci. Commun.,
2001, Jan. 3(1).

12. S. J. Pitts and B. Kratochvil, J. Forensic Sci., 1991, 36, 122.

13. K. W. Terry, A. V. Riessen, B. F. Lynch, and D. J. Vowles, Forensic Sci. Int., 1984, 25, 19.

14. R. D. Koons, C. A. Peters, and P. S. Rebbert, J. Anal. At. Spectrom., 1991, 6, 451

15. J. A. Buscaglia, Anal. Chim. Acta, 1994, 288, 17.

16. Y. Suzuki, R. Sugita, S. Suzuki, Y. Higashikawa, and Y. Marumo, Report of the National Research Institute of Police Science (Research on Forensic Science), 1995, 48, 7.

17. D. A. Hickman, Forensic Sci. Int., 1983, 23, 213.

18. R. D. Koons, C. Fiedler, and R. C. Rawalt, J. Forensic Sci., 1988, 31, 49 .

19. Y. Suzuki, R. Sugita, S.Suzuki, and Y. Marumo, Bunseki Kagaku, 1997, 46, 825.

20. Y. Suzuki, R. Sugita, S. Suzuki, and Y. Marumo, Anal. Sci., 2000, 16, 195

21. Y. Suzuki, M. Kasamatsu, R. Sugita, H. Ohta, S. Suzuki, and Y. Marumo, Bunseki Kagaku, 2003, 52, 469.

22. S. Montero, A. L. Hobbs, T. A. French, and J. R. Almirall, J. Forensic Sci., 2003, 48, 1101.

23. T. Trejos, S. Montero, and J. R. Almirall, Anal. Bioanal. Chem., 2003, 376, 1255 .

24. I. Nakai, Y. Terada, M. Itou, and Y. Sakurai, J. Synchrotron Radiat., 2001, 8, 49.

25. N. Kondo, N. Ota, R. I. Nakai, and A. Sasaki, Bunseki Kagaku, 1997, 46, 957.

26. M. Ezoe, M. Sasaki, A. Hokura, I. Nakai, Y. Terada, T. Yoshinaga, K. Tukamoto, and A. Hagiwara, Bunseki Kagaku, 2002, 51, 883.

27. J. A. Zoro, J. Locke, R. S. Day, O. Badmus, and A. C. Perrymann, Forensic Sci. Int., 1988, 39, 127.

28. H. Ida and J Kawai, Anal. Sci., 2004, 20, 1211 